\title{
Understanding Competition of Polyalcohol Dehydration Reactions in Hot Water
}

Yong Lik Chang, ${ }^{\dagger}$ Takehiko Sasaki, ${ }^{\dagger}$ Jordi Ribas-Ariño, ${ }^{\ddagger}$ Masahiko Machida, ${ }^{\complement}$ and Motoyuki Shiga*

$\dagger$ Department of Complexity Science and Engineering, Graduate School of Frontier Sciences, The University of Tokyo, 5-1-5, Kashiwanoha, Kashiwa 277-8561, Japan

$\ddagger$ Departament de Química-Física i CERQT, Universitat de Barcelona, Diagonal, 645, 08028-Barcelona, Spain

\Center for Computational Science and e-Systems, Japan Atomic Energy Agency, 178-4-4,

Wakashiba, Kashiwa, Chiba 277-0871, Japan.

E-mail: shiga.motoyuki@jaea.go.jp. 


\begin{abstract}
Dehydration of biomass-derived polyalcohols has recently drawn attention in green chemistry as a prototype of selective reactions controllable in hot water or hot carbonated water, without any use of organic solvents or metal catalysts. Here we report a free energy analysis based on first-principles metadynamics and blue-moon ensemble simulations to understand the mechanism of competing intramolecular dehydration reactions of 1,2,5-pentanetriol (PTO) in hot acidic water. The simulations consistently predict that the most dominant mechanism is the proton-assisted $\mathrm{S}_{\mathrm{N}} 2$ process where the protonation of the hydroxyl group by water and the C-O bond breaking and formation occurs in a single step. However the free energy barriers are different between the reaction paths: those leading to five membered ether products, tetrahydrofurfuryl alcohol (THFA), are few kcal/mol lower than those leading to six membered ether products, 3-hydroxytetrahydropyran (3-HTHP). A slight difference is seen in the timing of the protonation of the hydroxyl group of THFA and 3-HTHP on their reaction pathways. The detailed mechanism found from the simulations shows how the reaction paths are selective in hot water and why the reaction rates are accelerated in acidic environments, thus giving a clear explanation of experimental findings for a broad class of competing dehydration processes of polyalcohols.
\end{abstract}




\section{Introduction}

The development of green and sustainable processes for the production of chemicals and energy carriers is one of the most pressing challenges faced by our society. In this context, biomass conversion in hot pressurized water (be it either sub- or supercritical water) has recently emerged as a very promising technology. ${ }^{1-6}$ On the one hand, the use of renewable biomass resources associated with this technology can give rise to an environmentally beneficial reduction in the carbon footprint of chemicals and liquid fuel and can contribute to the development of a sustainable industrial society. ${ }^{7-10}$ In addition to this, the use of water as a reaction medium for organic processes is in line with green chemistry practices because water is non-toxic, non-flammable, inexpensive, environmentally benign and readily available. ${ }^{11}$ In contrast with the low solubility for many small organic molecules in room-temperature liquid water, the same compounds are much more soluble in liquid water above $\sim 200^{\circ} \mathrm{C}$ by virtue of a drastic decrease of the dielectric constant. ${ }^{12}$ Consequently, an increase in temperature of liquid water widens the scope of reactions that can be carried out, thus rendering both subcritical high-temperature liquid water (HTW, i.e. liquid water above $\sim 200^{\circ} \mathrm{C}$ ) and supercritical water very interesting reaction media for biomass conversion and organic synthesis reactions. $^{13-16}$

The use of hot pressurized water as a reaction medium, besides being aligned with green chemistry guidelines, is very appealing from the perspective of optimization of chemical processes because water temperature and water density or pressure can exert a strong influence on product selectivities and reaction rates. ${ }^{17-20}$ An in-depth knowledge of the reaction mechanism and the role played by water is clearly very beneficial when it comes to identifying the optimal conditions for a given reaction. Detailed knowledge on how the special properties of hot pressurized water affect reactivity is also very important in other research areas, such as geochemistry ${ }^{21,22}$ and origin of life. ${ }^{23,24}$ It can be very difficult to elucidate the role of hot pressurized water in reaction mechanisms exclusively from experiments because water can interact in many and complex ways with the reacting system (through hydrogen 
bonding, its polarity, its capacity of acting as an acid or basic catalyst, hydrophobic effects, etc.) and because reaction mechanisms operating in room-temperature water can drastically differ from those operating at high temperatures and pressures. ${ }^{25,26}$ In view of the proven utility of computational chemistry in establishing reaction mechanisms in room-temperature aqueous media, ${ }^{27-31}$ it is clear that computational studies of reactivity in hot pressurized water, which are still scarce ${ }^{25,26,32,33}$ in comparison with those at ambient temperature, can provide valuable insights into reaction mechanisms, thereby facilitating the design of optimal chemical processes in hydrothermal conditions. Here we elucidate the mechanism and the origin of selectivity of an intramolecular dehydration process that serves as a model system for polyalcohol dehydration reactions in hot pressurized water.

Among all processes relevant for biomass conversion, the dehydration of polyalcohol compounds is one of the most intensively investigated class of reactions. ${ }^{34-36}$ The use of $\mathrm{CO}_{2}$-enriched subcritical high temperature liquid water (HTW) has recently emerged as a very promising technology for polyalcohol dehydration ${ }^{37-44}$ and other biomass conversion or organic synthesis processes. ${ }^{45-47}$ The addition of $\mathrm{CO}_{2}$ to HTW leads to the formation of carbonic acid, which results in an acidic aqueous medium that is able to provide acid-catalyzed dehydration reactions. The use of $\mathrm{CO}_{2}$ instead of mineral acids as a means of providing an acid medium responds to environmental concerns associated with the latter type of acids.

The chemistry of intramolecular dehydration reactions of biomass-derived carbohydrates is very complex due to the large number of alcohol groups of these molecules and, thus, the large number of different products that can be formed. Taking this into consideration, studies on simpler model systems are very relevant because they can provide easier ways of establishing the underlying reaction mechanisms. One of the most prominent experimental studies on model systems for intramolecular dehydration reactions was reported by the research group led by Shirai a few years ago. ${ }^{39}$ In this study, 1,2,5-pentanetriol (PTO) was successfully converted into tetrahydrofurfuryl alcohol (THFA) and 3-hydroxytetrahydropyran (3-HTHP) by intramolecular dehydration in $\mathrm{HTW}$ at $573 \mathrm{~K}$ in the presence of $20 \mathrm{MPa}$ of $\mathrm{CO}_{2}$, as 
shown in Figure 1. This conversion was observed to be highly selective since the yield ratio of THFA to 3-HTHP was 7:1 in the total product mixture. This led to the conclusion that five-membered cyclic ethers were formed faster than six-membered cyclic ethers. ${ }^{39}$

In the present study, we carried out first principles molecular dynamics simulations in the condensed phase (under the same conditions as described in the experiment by Shirai and coworkers) combined with several enhanced sampling techniques with the goal of establishing the mechanism of PTO dehydration in HTW and elucidating the origin of its selectivity. It should be mentioned that the mechanism of dehydration reactions of dialcohols or glycerol in HTW has been investigated in previous computational works using different simulation techniques. ${ }^{32,48,49}$ However, this is the first computational work dealing with selectivity issues when both five-membered and six-membered cyclic ethers can be formed.

\section{Method}

Metadynamics $^{50}$ (MTD) simulations were used to study the free energy landscape of the aqueous PTO molecule with respect to the reactive regions toward the THFA and 3-HTHP products. Blue moon ensemble ${ }^{51}$ (BME) simulations were used to study free energy changes with respect to the non-reactive regions among the contracted and extended conformations of the aqueous PTO molecule. The simulations were conducted in the Born-Oppenheimer scheme, both for those based on first-principles density functional theory (DFT) and for those based on the semiempirical density functional tight binding (DFTB) method. We used the PIMD ${ }^{52}$ code in which MTD and BME methods are implemented in hierarchical parallelization linking to electronic structure codes, in this case, ${ }^{53}$ Vienna ab initio simulation package (VASP) ${ }^{54-57}$ and DFTB.$+{ }^{58-61}$

The MTD and BME simulations of the aqueous PTO molecule were carried out in the canonical (NVT) ensemble with constant volume corresponding to the pressure of 20 $\mathrm{MPa}$ and constant temperature of $573 \mathrm{~K}$, i.e., the same conditions as the previous exper- 
iment. ${ }^{39}$ The temperature was strongly controlled by massive Nosé-Hoover chain (MNHC) thermostats $^{62-64}$ for efficient statistical sampling. The simulations were executed in cubic boxes with periodic boundary conditions. The box sizes were determined from preliminary classical molecular dynamics simulations in the NPT ensemble at $20 \mathrm{MPa}$ and $573 \mathrm{~K}$ using OPLS force field. ${ }^{65}$ In order to mimic the experiment in acidic condition, we added one proton into the simulation box. This resulted in the aqueous solution being more acidic in the simulations $(\mathrm{pH}<1)$ than in the experiment $(\mathrm{pH} \sim 4)$, but this limitation cannot be avoided for molecular simulations taking account of water molecules explicitly. We note that the results of the current study on PTO dehydration with 30-70 water molecules should not be affected significantly by the system size since the reactions take place locally. In fact, in the case of hexanediol dehydration the free energy barrier in a microsolvated cluster with only six water molecules was found to have the same value (36 kcal/mol) as that in bulk water with 70 water molecules with periodic boundary conditions. ${ }^{32}$ Now we describe the simulation setup in detail. A summary is also shown in Table 1.

\section{DFT}

The DFT simulations were carried out for a system containing one PTO molecule, 30 water molecules and a proton. Uniform background charge was added to maintain the charge neutrality of the system. The electronic structure calculations were based on density functional theory with the PBE exchange correlation functional ${ }^{66}$ and Grimme's D2 van der Waals correction ${ }^{67}$ using a cut off at $400 \mathrm{eV}$ in the plane wave (PW) basis functions. The core electrons were taken into account using the projector augmented wave (PAW) method. ${ }^{68,69}$

To check the accuracy and validity of the PBE functional, a set of static calculations were performed based on the polarizable continuum model using a dielectric constant in hot water $\epsilon=25$, see SI. With respect to the relative energies for the confined/extended conformations of PTO and the relative energies for the 3-HTHP and THFA products in the respective optimized geometries (Figure S5), it was found that PBE functional exhibits the 
same qualitative trend as the calculation methods of higher accuracy such as B3LYP and MP2 (Table S1).

\section{DFTB}

The DFTB simulations were carried out for a system containing one PTO molecule, 70 water molecules, a proton and a chloride ion. In DFTB only the valence electrons are explicitly solved. The 3ob parameter set ${ }^{70,71}$ was used to describe the DFTB Hamiltonian.

\section{Metadynamics}

A series of three-dimensional metadynamics was carried out following a similar protocol proposed by Ensing et al. ${ }^{72}$ except that multiple walkers were used. ${ }^{73}$ The pseudo-Hamiltonian is

$$
H=H_{\mathrm{sys}}(\mathbf{r}, \mathbf{p})+\sum_{i, k} \frac{p_{i, k}^{2}}{2 \mu_{i}}+\sum_{i, k} \frac{\kappa_{i, k}}{2}\left(s_{i, k}-\tilde{s}_{i, k}(\mathbf{r})\right)^{2}+V_{\mathrm{hills}}(\mathbf{s}(t), t)+H_{\mathrm{mnhc}} .
$$

Here, the first term is the Hamiltonian of the molecular system given by the sum of kinetic energy of atoms and the Born-Oppenheimer potential calculated with either the DFT or DFTB method. The second term is the kinetic energy of fictitious particles (walkers) where $p_{i, k}$ and $\mu_{i}$ are the momentum and the mass of the walker, $i$ and $k$ being indices of the collective variable $(\mathrm{CV})$ component and the walker, respectively. The third term is a harmonic potential that binds the position of walker, $s_{i, k}$, to the actual CV value, $\tilde{s}_{i, k}(\mathbf{r})$, where $\kappa_{i, k}$ is the force constant. The fourth term is a history-dependent potential given by the sum of small Gaussian functions (hills) deposited to the places where all the walkers have visited until time $t$, which can be expressed as

$$
V_{\text {hills }}(\mathbf{s}(t), t)=\sum_{\tau<t} \sum_{k=1}^{N_{w}} h \exp \left[-\sum_{i=1}^{3} \frac{1}{2}\left(\frac{s_{i, k}(t)-s_{i, k}(\tau)}{\sigma_{i}}\right)^{2}\right]
$$


where $h$ and $\sigma_{i}$ are the hill height and the hill width, respectively, and $N_{w}$ is the number of walkers. The fifth term is the contribution from the MNHC thermostats. The free energy surface is estimated by

$$
A(\mathbf{s}) \approx-V_{\text {hills }}(\mathbf{s})+\text { const. }
$$

at a certain time when the walkers escaped from the PTO reactant basin (in the region of $d<0$, see below).

In the MTD simulations, three CVs, $s_{1}=d, s_{2}=\phi$ and $s_{3}=n$, were chosen in the same manner as the previous study of aqueous hexanediol. ${ }^{32}$ These CVs are selected based on the results of the minimum energy path calculations of microsolvated model of hexanediol; $d$ is the difference in bond distances of the two $\mathrm{C}-\mathrm{O}$ bonds that represent the exchange of bond breaking and formation upon the ether ring formation, $d=r_{\mathrm{CO}}-r_{\mathrm{OC}}, \phi$ is an O-CC-C dihedral angle which characterizes the conformation of the PTO molecule, and $n$ is the hydrogen coordination number for one of the alcohol oxygen atoms, $i$, which is expressed as a sum of rational functions,

$$
n=n_{i}=\sum_{j \in \mathrm{H}} \frac{1-\left(\frac{r_{i j}}{r_{\mathrm{b}}}\right)^{6}}{1-\left(\frac{r_{i j}}{r_{\mathrm{b}}}\right)^{12}},
$$

where $r_{\mathrm{b}}=1.4 \AA$ is a parameter to identify $\mathrm{OH}$ bonds. However, the atom numbers relevant to the CVs should be chosen differently for the respective reaction paths, see Figure 1 . The atoms associated to the CVs for each reaction path are listed in Table 2.

We used $N_{w}=12$ walkers to speed up the calculation. The force constants were set as $\left(\kappa_{1}, \kappa_{2}, \kappa_{3}\right)=\left(1191 \mathrm{kcal} \mathrm{mol}^{-1} \AA^{-2}, 630 \mathrm{kcal} \mathrm{mol}^{-1}, 630 \times 10^{-4} \mathrm{kcal} \mathrm{mol}^{-1} \mathrm{deg}^{-2}\right)$ which is large enough to strongly bind the walkers to the actual CV positions. The hill size was diminished to $\left(h, \sigma_{1}, \sigma_{2}, \sigma_{3}\right)=\left(0.2 \mathrm{kcal} / \mathrm{mol}, 0.08 \AA, 0.033,9^{\circ}\right)$. A new hill was deposited every time $(\tau)$ when a walker was displaced by $1.5|\boldsymbol{\sigma}|$. The fictitious mass was

$$
\mu_{i}=k_{B} T\left(\frac{\tau}{1.5 \sigma_{i}}\right)
$$


where $\tau(=10 \mathrm{fs})$ is the characteristic time for fictitious particle to move 1.5 times the hill width and $\sigma_{i}$ is the hill width of CV. With this setup, the diffusibility of the walkers was controlled such that the average interval of hill deposition was 10.6 fs. The slow rate at which the hills were deposited ensures a sufficiently long simulation and, thus, a correct mapping of the free energy surfaces associated with the reactions, which gives access to the thermodynamically preferred reaction pathways and properly evaluated activation free energies.

To explore the free energy surface of the reactive region only $(d<2.38 \AA)$, an artificial wall potential was added to $V_{\text {hills }}$. With this prescription we were able to prevent the computation of MTD simulations from being too expensive and from being unstable in statistics. However, the conformational change of PTO molecule was not fully covered within $d<2.38$ A. As shown below, the contracted conformations were in this range while the extended conformation was not. To remove this artifact, we used the blue moon ensemble simulation which is described next.

\section{Blue moon ensemble}

The blue moon ensemble simulations were used to compute the free energy difference with respect to the conformational change of PTO within the range $1.59 \AA \leq d \leq 5.11 \AA$. The reactive region was not studied in the blue moon ensemble simulations because the reaction cannot be described by a single dimension with respect to $d$.

The pseudo-Hamiltonian of blue moon ensemble, in which the system is constrained to $d=\tilde{s}(\mathbf{r})$, can be expressed as

$$
H_{\alpha}=H_{\mathrm{sys}}(\mathbf{r}, \mathbf{p})+\frac{\kappa}{2}(s-\tilde{s}(\mathbf{r}))^{2}+H_{\mathrm{mnhc}}
$$

where the force constant was set to a large value, $\kappa=5600 \mathrm{kcal} / \mathrm{mol} \AA^{-2}$, so as to keep the actual $\mathrm{CV}, \tilde{s}(\mathbf{r})$, close to the target value, $d$. The free energy value at $d=d_{\alpha}$, was evaluated 
as a numerical integration by the trapezoidal rule

$$
\begin{aligned}
A\left(d_{\beta}\right) & =A\left(d_{1}\right)+\int_{d_{1}}^{d_{\beta}}\left(\frac{\partial A}{\partial s}\right) d s \\
& \approx A\left(d_{1}\right)+\sum_{\alpha=2}^{\beta} \frac{1}{2}\left[\left\langle\kappa\left(d_{\alpha}-\tilde{s}\right)\right\rangle_{\alpha}+\left\langle\kappa\left(d_{\alpha-1}-\tilde{s}\right)\right\rangle_{\alpha-1}\right]\left(d_{\alpha}-d_{\alpha-1}\right)
\end{aligned}
$$

where the thermal averages $\langle\cdots\rangle_{\alpha}$ are computed at constraint points of $1 \leq \alpha \leq 12$ with equal separations, $d_{\alpha}=1.59 \AA+(\alpha-1) \times 0.32 \AA$.

\section{Results and discussion}

\section{Metadynamics}

Three-dimensional free energy profiles from DFT metadynamics are displayed in Figures 2 and 3 for the pathways leading to THFA (5PTO2 and 5PTO5) and 3-HTHP (6PTO1 and 6PTO5), respectively. (The corresponding results from DFTB metadynamics are also shown in Figure S1 and S2, respectively.) Each of these free energy profiles was obtained from the statistical average of four MTD runs. Gaining sufficient statistics helped us obtain qualitatively correct features of the free energy surfaces, although some roughness remains as an intrinsic error from the metadynamics sampling. (We will show that the errors of the reaction barrier later in Table 3 are about a few $\mathrm{kcal} / \mathrm{mol}$.)

In Figures 2 and 3 we find the free energy minima in the regions in black, with the difference in bond distance $d \simeq 1.9 \AA$, the hydrogen coordination number $n \simeq 1.2$, and the dihedral angles $\phi \simeq 60^{\circ}, 180^{\circ}$, and $300^{\circ}$. These values correspond to an ensemble of contracted conformations of PTO molecule with neutral hydroxyl group (the value of $n$ is greater than 1.0 because the hydroxyl group creates hydrogen bonds with water). As time evolves the MTD simulation explores from the lower free energy regions to the higher free energy regions in orange and then in yellow. Finally the MTD runs were terminated when 
we observe an escape from the CV range $(d<0)$ after visiting the highest free energy region in grey. The $\mathrm{C} \cdots \mathrm{O}-\mathrm{C} \rightarrow \mathrm{C}-\mathrm{O} \cdots \mathrm{C}$ bond exchange occurs to form a protonated ether upon crossing the $d=0$ line. After that, we observed a quick proton release to form the ether products, i.e. THFA in the case of Figure 2 and and 3-HTHP in the case of Figure 3. Thus, $d=0$ can be regarded as the transition state of these reactions.

At the transition state (when crossing the $d=0$ line), we can see that $n \simeq 2.0$, which means that one of the hydroxyl groups is always protonated, and $\phi$ is in between $180^{\circ}$ and $270^{\circ}$, which means that the backbone of PTO molecule is always in a specific conformation. The protonation of the hydroxyl group in the process is in line with experimental evidence that the reaction rate is accelerated in acidic conditions by carbonated HTW. ${ }^{39}$ Inspecting the trajectory, we find that the PTO conformation of $180^{\circ}<\phi<270^{\circ}$ allows the alignment of $\mathrm{O} \cdots \mathrm{C}-\mathrm{O}$ atoms to enable the bond exchange via an $\mathrm{S}_{\mathrm{N}} 2$ process. We did not find any free energy minima in the protonated region $(n \sim 2)$ before the bond exchange $(d>0)$. This means there are no stable protonated intermediates of PTO, and thus the protonation and bond exchange proceeds consecutively in a single step. All four paths found in this study, i.e., 5PTO2, 5PTO5, 6PTO1 and 6PTO5, share such a proton assisted $\mathrm{S}_{\mathrm{N}} 2$ mechanism. This is also similar to what was found earlier for the dehydration reaction of hexanediol. ${ }^{32}$ Therefore, this mechanism should be general for polyalcohol dehydration in HTW under acidic conditions.

In Figures 5 and 6, we show the molecular configurations along the 5PTO2 and 6PTO5 pathways. (The molecular configurations along the 5PTO5 and 6PTO1 pathways are also shown in Figures S6 and S7, respectively.) As shown in Figures 5 and 6, the alcohol oxygen atom $\left(\mathrm{O}_{7}\right.$ in $5 \mathrm{PTO} 2, \mathrm{O}_{8}$ in $\left.6 \mathrm{PTO} 5\right)$ of $\mathrm{PTO}$ was protonated by its surrounding water molecules. Then, the alcohol oxygen atom $\left(\mathrm{O}_{8}\right.$ in 5PTO2, $\mathrm{O}_{6}$ in 6PTO5) of the opposite side attacked the carbon $\left(\mathrm{C}_{2}\right.$ in $5 \mathrm{PTO} 2, \mathrm{C}_{5}$ in $\left.6 \mathrm{PTO} 5\right)$ and in the process released the protonated hydroxide as a water molecule. In 5PTO2, the carbon $\left(\mathrm{C}_{2}\right)$ attacked by the oxygen atom is the stereocenter of PTO, and thus the dehydration of $R$-PTO yields $S$-THFA. The hydrogen 
atom which was still attached to the oxygen atom of the ether ring was later taken away by the surrounding water molecules to form THFA and 3-HTHP, respectively. Both the proton acceptance from and the proton release occurred through hydrogen bonded network of water molecules via the Grotthuss mechanism.

\section{Blue moon ensemble}

In Figure 7, we show the free energy profile along $d$, the difference in $\mathrm{C}-\mathrm{O}$ and $\mathrm{O}-\mathrm{C}$ distances, which is obtained from the DFT blue moon ensemble simulations. (The corresponding results from the DFTB blue moon ensemble simulations are also shown in Figure S3.) The shallow shoulder shown at $d \simeq 1.9 \AA$ are the confined conformations, which correspond to the minima in Figure 5 and 6, respectively. However, we find that there is a more stable minimum; although they have different $d$ values depending on the pathways, they are of the same conformation - the extended conformation of PTO. Therefore, the extended conformation is the most stable state of PTO in the aqueous solution in thermal equilibrium $(573 \mathrm{~K}, 20$ $\mathrm{MPa}$ ). In Figure 7, the origin of the free energy is set to that of the extended conformation.

The free energy changes due to the conformational change are of few kcal/mol, which is smaller than those of the bond breaking by an order of magnitude. However, we find that the free energy change from the extended to the confined conformations does depend on the reaction paths. Since the free energy change corresponds to the reversible work to fold the conformation, the energy change tends to increase as the folding stride, $\Delta d$, increases. Accordingly, the free energy in the confined conformations along the paths leading to 3HTHP is larger than those of THFA. The dependence on the respective paths varies from 0.9 to $4.0 \mathrm{kcal} / \mathrm{mol}$, which means that the free energy correction due to the conformational change cannot be neglected for correct estimation of reaction barriers. 


\section{Reaction Barrier}

The free energy barrier of each reaction path, along with the contributions from metadynamics and blue moon ensemble simulations are summarized in Table 3. The contributions from MTD simulations, $\Delta A_{\mathrm{MTD}}$, are estimated as the difference of free energies at $d=0.0 \AA$ (corresponding to the transition state of the reactions) and $d=2.38 \AA$ (the confined PTO), taken from the free energy profile in Figures 2 and 3, respectively. The contributions from blue moon simulations, $\Delta A_{\mathrm{BME}}$, are estimated as the difference free energies at $d=2.38$ $\AA$ (corresponding to the confined PTO) and $d_{\text {min }}$ (the free energy minimum, the extended PTO), taken from the free energy curves in Figure 7. The total free energy barrier, $\Delta A_{\text {total }}$ is estimated as the sum of $\Delta A_{\mathrm{MTD}}$ and $\Delta A_{\mathrm{BME}}$.

Estimates of free energy barriers from experiments were based on the half life of PTO $\left(\tau_{\text {exptl }}=30\right.$ minutes $)$ and the product ratio $\left(x_{\text {exptl }}=1 / 8\right.$ for 3 -HTHP and $x_{\text {exptl }}=7 / 8$ for THFA). ${ }^{39}$ The measured rate constant

$$
k_{\text {exptl }}=\frac{x_{\text {exptl }}}{\tau_{\text {exptl }}} \ln 2
$$

was assumed to be equal to the rate constant from the transition state theory (TST),

$$
k_{\text {exptl }}=\frac{k_{\mathrm{B}} T}{h} \exp \left(-\frac{\Delta A_{\text {exptl }}}{k_{\mathrm{B}} T}\right) \text {, }
$$

where $k_{\mathrm{B}}$ is the Boltzmann constant, $h$ is the Planck constant, and $T$ is the temperature, $573 \mathrm{~K}$. The free energy barriers thus estimated were $\Delta A_{\text {exptl }}=41.7$ and $43.9 \mathrm{kcal} / \mathrm{mol}$, respectively, for THFA and 3-HTHP.

The computed free energy barriers for the reaction path leading to THFA $(33 \mathrm{kcal} / \mathrm{mol}$ for DFT and 33-35 kcal/mol for DFTB) are lower than those leading to 3-HTHP (35-36 $\mathrm{kcal} / \mathrm{mol}$ for DFT and 37-43 kcal/mol for DFTB). Although the computed free energies are systematically lower than the experimental ones, both the results of DFT and DFTB 
support the experimental finding that THFA and 3-HTHP are the main and by-products, respectively. The difference between the computed free energy barriers between THFA and 3-HTHP products are $6 \mathrm{kcal} / \mathrm{mol}$ in DFT and $4 \mathrm{kcal} / \mathrm{mol}$ in DFTB, which are larger than those estimated from experiment, $2.2 \mathrm{kcal} / \mathrm{mol}$. Possible origins for the discrepancy between the simulation and the experiment are multiple; it could be ascribed to the DFT and DFTB methods, the high $\mathrm{pH}$ condition in the computation, or the experimental estimation assuming the TST.

Still, the simulations provide some insights on a qualitative basis. It is estimated that the free energy barriers of two paths leading to THFA (5PTO2 and 5PTO5) are close to each other, 33 and 33-35 kcal/mol in DFT and DFTB, respectively. This suggests that reaction paths 5PTO2 and 5PTO5, which lead to $S$-THFA and $R$-THFA, are almost equally probable. From this result it is predicted that the main product THFA would be a racemic mixture. Meanwhile, the paths leading to 3-HTHP (6PTO1 and 6PTO5) are both stereoselective since they were $\mathrm{S}_{\mathrm{N}} 2$ reactions. Therefore, for a 3 -HTHP pathway, S-PTO leads to $S$-3-HTHP and $R$-PTO leads to $R$-3-HTHP. In fact, our previous studies on hexanediol (without the $3-\mathrm{OH}$ ) have shown that the reaction is stereoselective. ${ }^{32}$

\section{Reaction Path Analysis}

To understand the origin of the difference in free energy barriers, the minimum free energy paths (MFEPs) were analyzed. The MFEPs were obtained in the following manner from the free energy surfaces of DFT metadynamics simulations. The three-dimensional free energy surfaces, which are shown in Figures 2 and 3, were cut into slices with constant $d$ values. Within two-dimensional free energy surface of each slice, we found the point $\left(n_{\mathrm{mfe}}(d), \phi_{\mathrm{mfe}}(d)\right)$ where the free energy $A_{\text {mfe }}(d)$ becomes a minimum. The physical meaning of the free energy minimum is the most probable state, in this case, among the states from the canonical ensemble of molecular configurations. Therefore, given a $d$ value, $\left(n_{\mathrm{mfe}}(d), \phi_{\mathrm{mfe}}(d)\right)$ are the most probable $(n, \phi)$ values that are found in the canonical ensemble. 
In Figure $8(\mathrm{a})$, we display the free energy curves $A_{\mathrm{mfe}}(d)$ along the MFEP, together with the free energy curves obtained from MTD and BME in the range $0 \AA<d<5.1$ $\AA$. The origin of these curves are aligned in the extended conformation of PTO. We can see that the free energy curves leading to THFA and 3-HTHP start to deviate below the range $d \simeq 1.7 \AA$ and above $A \simeq 10 \mathrm{kcal} / \mathrm{mol}$. In Figure $8(\mathrm{~b})$, we display the plots of the coordination number along the MFEP $n_{\text {mfe }}(d)$ and $A_{\text {mfe }}(d)$. We find that the plots are very different between the MFEP leading to THFA and 3-HTHP above $A \simeq 10 \mathrm{kcal} / \mathrm{mol}$; the most probable of the protonation occurs at $A \simeq 10 \mathrm{kcal} / \mathrm{mol}$ and $A \simeq 30 \mathrm{kcal} / \mathrm{mol}$ in the cases of THFA and 3-HTHP reactions, respectively. Therefore, from the MFEP analysis we speculate that the timing of protonation before the C-O bond breaking and formation process may be causing the difference in the free energy barrier of the reaction leading to THFA and 3-HTHP. (In contrast, the MFEP of the DFTB calculation did not show much difference between the THFA and 3-HTHP pathways, see Figure S4; this may be ascribed to the failure of DFTB method to describe properly the change in proton affinities of PTO at different configurations.)

\section{Conclusions}

The competing reactions of PTO dehydration in HTW were studied by MTD and BME simulations. The results obtained from the DFT- and DFTB-based simulations indicate consistently that the free energy barriers leading to the five membered ether product, THFA, are lower than those leading to the six membered ether product, 3-HTHP. This is in accordance with experimental evidence that THFA and 3-HTHP are the main product and the byproduct respectively. It was found that the proton-assisted $\mathrm{S}_{\mathrm{N}} 2$ process, in which the protonation of hydroxyl group and the C-O bond exchange occurs in a single step, is the most dominant mechanism. As this process was seen in all these four reaction paths studied, it is expected that this is common in polyalcohol dehydration in HTW under acidic conditions. The sim- 
ulations clarified the importance of hydrogen bond network of water enabling fast proton transfer such that it facilitates the protonation of hydroxyl group. This clearly explains why the reaction is accelerated in carbonated HTW. From the minimum free energy path analysis of DFT-based simulations, it was found that the reaction mechanism were slightly different between THFA and 3-HTHP reactions in the timing of the protonation of hydroxyl group, which could be a source of difference in their reaction rates.

The simulation predicts that the free energy barriers of the two $\mathrm{S}_{\mathrm{N}} 2$ reaction paths from $S$-PTO leading to $R$ - and $S$-TFHA are similar. Thus it is expected that the TFHA product should be racemized. On the other hand, S-PTO should mostly lead to $S$-3-HTHP since the stereoselectivity is kept in the two $\mathrm{S}_{\mathrm{N}} 2$ reaction paths.

The results obtained in this work rely on the MTD simulations which detect a reaction path with lowest free energy barrier for a given range of CV space. Nothing is said so far about the possibility of the reactive process leading to a minor product which should, basically, experience a reaction path overcoming a higher free energy barrier. In fact, experiments found a deterioration of selectivity with minor products, such as polymers that arise presumably via inter-molecular dehydration. One of the next challenges in theoretical studies of polyalcohol dehydration in water would be to identify the minor product and understand the reaction mechanism leading to minor products.

\section{Acknowledgement}

The computations in this work were mostly done using the supercomputer facilities at Japan Atomic Energy Agency and the Institute for Solid State Physics, the University of Tokyo. M. S. thanks support from JSPS KAKENHI Grant Numbers 16K05675 and 18H05519. J. R. A. acknowledges the Spanish Government for project CTQ2017-87773-P/AEI/FEDER, UE (MICINN) and Catalan DURSI for project 2017SGR348. The authors thank Dr. Sergi Ruiz Barragan at University of Bochum for useful discussion. The authors are grateful to 
Dr. Alex Malins at JAEA for proofreading the manuscript.

\section{References}

(1) Yu, Y.; Lou, X.; Wu, H. Some recent advances in hydrolysis of biomass in hotcompressed water and its comparisons with other hydrolysis methods. Energy Fuels 2008, 22, 46-60.

(2) Toor, S. S.; Rosendahl, L.; Rudolf, A. Hydrothermal liquefaction of biomass: A review of subcritical water technologies. Energy 2011, 36, 2328-2342.

(3) Yeh, T. M.; Dickinson, J. G.; Franck, A.; Linic, S.; Thompson Jr, L. T.; Savage, P. E. Hydrothermal catalytic production of fuels and chemicals from aquatic biomass. $J$. Chem. Technol. Biotechnol. 2013, 88, 13-24.

(4) Kruse, A.; Dahmen, N. Water-A magic solvent for biomass conversion. J. Supercrit. Fluids 2015, 96, 36-45.

(5) Knez, Ž.; Markočič, E.; Hrnčič, M. K.; Ravber, M.; Škerget, M. High pressure water reforming of biomass for energy and chemicals: A short review. J. Supercrit. Fluids 2015, 96, 46-52.

(6) Elliott, D. C.; Biller, P.; Ross, A. B.; Schmidt, A. J.; Jones, S. B. Hydrothermal liquefaction of biomass: Developments from batch to continuous process. Bioresour. Technol. 2015, 178, 147-156.

(7) Ragauskas, A. J.; Williams, C. K.; Davison, B. H.; Britovsek, G.; Cairney, J.; Eckert, C. A.; Frederick, W. J.; Hallett, J. P.; Leak, D. J.; Liotta, C. L. et al. The path forward for biofuels and biomaterials. Science 2006, 311, 484-489.

(8) Corma, A.; Iborra, S.; Velty, A. Chemical routes for the transformation of biomass into chemicals. Chem. Rev. 2007, 107, 2411-2502. 
(9) Sheldon, R. A. Green and sustainable manufacture of chemicals from biomass: State of the art. Green Chem. 2014, 16, 950-963.

(10) Fárran, A.; Cai, C.; Sandoval, M.; Xu, Y.; Liu, J.; Hernáiz, M. J.; Linhardt, R. J. Green solvents in carbohydrate chemistry: From raw materials to fine chemicals. Chem. Rev. 2015, 115, 6811-6853.

(11) Simon, M.-O.; Li, C.-J. Green chemistry oriented organic synthesis in water. Chem. Soc. Rev. 2012, 41, 1415-1427.

(12) Dunn, J. B.; Urquhart, D. I.; Savage, P. E. Terephthlic acid synthesis in supercritical water. Adv. Synth. Catal. 2002, 344, 385-392.

(13) Akiya, N.; Savage, P. E. Roles of water for chemical reactions in high-temperature water. Chem. Rev. 2002, 102, 2725-2750.

(14) Hunter, S. E.; Savage, P. E. Recent advances in acid-and base-catalyzed organic synthesis in high-temperature liquid water. Chem. Eng. Sci. 2004, 59, 4903-4909.

(15) Li, C.-J. Organic reactions in aqueous media with a focus on carbon-carbon bond formations: A decade update. Chem. Rev. 2005, 105, 3095-3166.

(16) Kruse, A.; Dinjus, E. Hot compressed water as reaction medium and reactant: Properties and synthesis reactions. J. Supercrit. Fluids 2007, 39, 362-380.

(17) Chandler, K.; Liotta, C. L.; Eckert, C. A.; Schiraldi, D. Tuning alkylation reactions with temperature in near-critical water. AIChE J. 1998, 44, 2080-2087.

(18) Ikushima, Y.; Hatakeda, K.; Sato, M.; Sato, O.; Arai, M. Innovation in a chemical reaction process using a supercritical water microreaction system: Environmentally friendly production of $\varepsilon$-caprolactam. Chem. Commun. 2002, 2208-2209.

(19) Akiya, N.; Savage, P. E. Kinetics and mechanism of cyclohexanol dehydration in hightemperature water. Ind. Eng. Chem. Res. 2001, 40, 1822-1831. 
(20) Nagai, Y.; Matubayasi, N.; Nakahara, M. Hot water induces an acid-catalyzed reaction in its undissociated form. Bull. Chem. Soc. Japan 2004, 77, 691-697.

(21) Siskin, M.; Katritzky, A. R. Reactivity of organic compounds in hot water: Geochemical and technological implications. Science 1991, 254, 231-237.

(22) Shipp, J.; Gould, I. R.; Herckes, P.; Shock, E. L.; Williams, L. B.; Hartnett, H. E. Organic functional group transformations in water at elevated temperature and pressure: Reversibility, reactivity, and mechanisms. Geochim. Cosmochim. Acta 2013, 104, 194-209.

(23) Huber, C.; Wächtershäuser, G. Activated acetic acid by carbon fixation on (Fe, Ni) S under primordial conditions. Science 1997, 276, 245-247.

(24) Shock, E. L.; McCollom, T.; Schulte, M. D. Geochemical constraints on chemolithoautotrophic reactions in hydrothermal systems. Origins Life Evol. Biosphere 1995, 25, 141-159.

(25) Nair, N. N.; Schreiner, E.; Marx, D. Peptide synthesis in aqueous environments: The role of extreme conditions on amino acid activation. J. Am. Chem. Soc. 2008, 130, 14148-14160.

(26) Schreiner, E.; Nair, N. N.; Marx, D. Peptide synthesis in aqueous environments: The role of extreme conditions on peptide bond formation and peptide hydrolysis. J. Am. Chem. Soc. 2009, 131, 13668-13675.

(27) Acevedo, O.; Armacost, K. Claisen rearrangements: Insight into solvent effects and “on water” reactivity from QM/MM simulations. J. Am. Chem. Soc. 2010, 132, 1966-1975.

(28) Thomas, L. L.; Tirado-Rives, J.; Jorgensen, W. L. Quantum mechanical/molecular mechanical modeling finds Diels-Alder reactions are accelerated less on the surface of water than in water. J. Am. Chem. Soc. 2010, 132, 3097-3104. 
(29) Hatanaka, M.; Morokuma, K. Role of water in Mukaiyama-aldol reaction catalyzed by lanthanide Lewis acid: A computational study. J. Am. Chem. Soc. 2013, 135, 1397213979.

(30) Zhang, J.; Yang, Y. I.; Yang, L.; Gao, Y. Q. Dynamics and kinetics study of “ InWater" chemical reactions by enhanced sampling of reactive trajectories. J. Phys. Chem. B 2015, 119, 14505-14514.

(31) Ribas-Arino, J.; Carvajal, M. A.; Chaumont, A.; Masia, M. Unraveling the role of water in the stereoselective step of aqueous proline-catalyzed aldol reactions. Chem. Eur. J. 2012, 18, 15868-15874.

(32) Ruiz-Barragan, S.; Ariño, J. R.; Shiga, M. The reaction mechanism of polyalcohol dehydration in hot pressurized water. Phys. Chem. Chem. Phys. 2016, 18, 3243832447 .

(33) Takahashi, H.; Hashimoto, H.; Nitta, T. Quantum mechanical/molecular mechanical studies of a novel reaction catalyzed by proton transfers in ambient and supercritical states of water. J. Chem. Phys. 2003, 119, 7964-7971.

(34) Román-Leshkov, Y.; Barrett, C. J.; Liu, Z. Y.; Dumesic, J. A. Production of dimethylfuran for liquid fuels from biomass-derived carbohydrates. Nature 2007, 447, 982.

(35) Haider, M. H.; Dummer, N. F.; Knight, D. W.; Jenkins, R. L.; Howard, M.; Moulijn, J.; Taylor, S. H.; Hutchings, G. J. Efficient green methanol synthesis from glycerol. Nat. Chem. 2015, \%, 1028-1032.

(36) Sun, D.; Yamada, Y.; Sato, S.; Ueda, W. Glycerol as a potential renewable raw material for acrylic acid production. Green Chem. 2017, 19, 3186-3213.

(37) Hunter, S. E.; Savage, P. E. Acid-catalyzed reactions in carbon dioxide-enriched hightemperature liquid water. Ind. Eng. Chem. Res. 2003, 42, 290-294. 
(38) Hunter, S. E.; Ehrenberger, C. E.; Savage, P. E. Kinetics and mechanism of tetrahydrofuran synthesis via 1,4-butanediol dehydration in high-temperature water. J. Org. Chem. 2006, 71, 6229-6239.

(39) Yamaguchi, A.; Hiyoshi, N.; Sato, O.; Bando, K. K.; Shirai, M. Enhancement of cyclic ether formation from polyalcohol compounds in high temperature liquid water by high pressure carbon dioxide. Green Chem. 2009, 11, 48-52.

(40) Yamaguchi, A.; Hiyoshi, N.; Sato, O.; Shirai, M. Sorbitol dehydration in high temperature liquid water. Green Chem. 2011, 13, 873-881.

(41) Yamaguchi, A.; Hiyoshi, N.; Sato, O.; Shirai, M. Cyclization of alkanediols in hightemperature liquid water with high-pressure carbon dioxide. Catal. Today 2012, 185, 302-305.

(42) Yamaguchi, A.; Hiyoshi, N.; Sato, O.; Shirai, M. Stereoselective intramolecular dehydration of 2,5-hexanediol in high-temperature liquid water with high-pressure carbon dioxide. ACS Catal. 2010, 1, 67-69.

(43) Sato, O.; Yamaguchi, A.; Shirai, M. Continuous dehydration of 1,4-butanediol in flowing liquid water with carbon dioxide. Catal. Commun. 2015, 68, 6-10.

(44) Yamaguchi, A.; Muramatsu, N.; Mimura, N.; Shirai, M.; Sato, O. Intramolecular dehydration of biomass-derived sugar alcohols in high-temperature water. Phys. Chem. Chem. Phys. 2017, 19, 2714-2722.

(45) Hunter, S. E.; Savage, P. E. Quantifying rate enhancements for acid catalysis in CO2enriched high-temperature water. AIChE J. 2008, 54, 516-528.

(46) Morais, A. R.; da Costa Lopes, A. M.; Bogel-Łukasik, R. Carbon dioxide in biomass processing: Contributions to the green biorefinery concept. Chem. Rev. 2014, 115, $3-27$. 
(47) Pigaleva, M. A.; Elmanovich, I. V.; Kononevich, Y. N.; Gallyamov, M. O.; Muzafarov, A. M. A biphase $\mathrm{H} 2 \mathrm{O} / \mathrm{CO} 2$ system as a versatile reaction medium for organic synthesis. RSC Adv. 2015, 5, 103573-103608.

(48) Takahashi, H.; Miki, F.; Ohno, H.; Kishi, R.; Ohta, S.; Furukawa, S.-i.; Nakano, M. Hydration effects on the reaction with an open-shell transition state: QM/MM-ER study for the dehydration reaction of alcohol in hot water. J. Math. Chem. 2009, 46, 781-794.

(49) Caratzoulas, S.; Courtney, T.; Vlachos, D. G. Hybrid quantum mechanics/molecular mechanics-based molecular dynamics simulation of acid-catalyzed dehydration of polyols in liquid water. J. Phys. Chem. A 2011, 115, 8816-8821.

(50) Laio, A.; Parrinello, M. Escaping free-energy minima. Proc. Natl. Acad. Sci. U.S.A. 2002, 99, 12562-12566.

(51) Carter, E.; Ciccotti, G.; Hynes, J. T.; Kapral, R. Constrained reaction coordinate dynamics for the simulation of rare events. Chem. Phys. Lett. 1989, 156, 472-477.

(52) PIMD version 2.1.1. https://ccse.jaea.go.jp/ja/download/pimd/index.en.html.

(53) Ruiz-Barragan, S.; Ishimura, K.; Shiga, M. On the hierarchical parallelization of ab initio simulations. Chem. Phys. Lett. 2016, 646, 130-135.

(54) Kresse, G.; Hafner, J. Ab initio molecular dynamics for liquid metals. Phys. Rev. B 1993, 47, 558-561.

(55) Kresse, G.; Hafner, J. Ab initio molecular-dynamics simulation of the liquid-metalamorphous-semiconductor transition in germanium. Phys. Rev. B 1994, 49, 1425114269.

(56) Kresse, G.; Furthmüller, J. Efficiency of ab-initio total energy calculations for metals and semiconductors using a plane-wave basis set. Comput. Mater. Sci. 1996, 6, 15-50. 
(57) Kresse, G.; Furthmüller, J. Efficient iterative schemes for ab initio total-energy calculations using a plane-wave basis set. Phys. Rev. B 1996, 54, 11169-11186.

(58) Porezag, D.; Frauenheim, T.; Köhler, T.; Seifert, G.; Kaschner, R. Construction of tight-binding-like potentials on the basis of density-functional theory: Application to carbon. Phys. Rev. B 1995, 51, 12947-12957.

(59) Seifert, G.; Porezag, D.; Frauenheim, T. Calculations of molecules, clusters, and solids with a simplified LCAO-DFT-LDA scheme. Int. J. Quantum Chem. 1996, 58, 185-192.

(60) Elstner, M.; Porezag, D.; Jungnickel, G.; Elsner, J.; Haugk, M.; Frauenheim, T.; Suhai, S.; Seifert, G. Self-consistent-charge density-functional tight-binding method for simulations of complex materials properties. Phys. Rev. B 1998, 58, 7260-7268.

(61) Aradi, B.; Hourahine, B.; Frauenheim, T. DFTB+, A sparse matrix-based implementation of the DFTB method. J. Phys. Chem. A 2007, 111, 5678-5684.

(62) Nosé, S. A unified formulation of the constant temperature molecular dynamics methods. J. Chem. Phys. 1984, 81, 511-519.

(63) Hoover, W. G. Canonical dynamics: Equilibrium phase-space distributions. Phys. Rev. A 1985, 31, 1695-1697.

(64) Martyna, G. J.; Klein, M. L.; Tuckerman, M. E. Nosé-Hoover chains: The canonical ensemble via continuous dynamics. J. Chem. Phys. 1992, 97, 2635-2643.

(65) Jorgensen, W. L.; Maxwell, D. S.; Tirado-Rives, J. Development and testing of the OPLS all-atom force field on conformational energetics and properties of organic liquids. J. Am. Chem. Soc. 1996, 118, 11225-11236.

(66) Perdew, J. P.; Burke, K.; Ernzerhof, M. Generalized gradient approximation made simple. Phys. Rev. Lett. 1996, r7, 3865-3868. 
(67) Grimme, S. Semiempirical GGA-type density functional constructed with a long-range dispersion correction. J. Comput. Chem. 2006, 27, 1787-1799.

(68) Blöchl, P. E. Projector augmented-wave method. Phys. Rev. B 1994, 50, 17953-17979.

(69) Kresse, G.; Joubert, D. From ultrasoft pseudopotentials to the projector augmentedwave method. Phys. Rev. B 1999, 59, 1758-1775.

(70) Gaus, M.; Goez, A.; Elstner, M. Parametrization and benchmark of DFTB3 for organic molecules. J. Chem. Theory Comput. 2012, 9, 338-354.

(71) Kubillus, M.; Kubar, T.; Gaus, M.; Rezac, J.; Elstner, M. Parameterization of the DFTB3 method for Br, Ca, Cl, F, I, K, and Na in organic and biological systems. $J$. Chem. Theory Comput. 2014, 11, 332-342.

(72) Ensing, B.; Laio, A.; Parrinello, M.; Klein, M. L. A recipe for the computation of the free energy barrier and the lowest free energy path of concerted reactions. J. Phys. Chem. B 2005, 109, 6676-6687.

(73) Raiteri, P.; Laio, A.; Gervasio, F. L.; Micheletti, C.; Parrinello, M. Efficient reconstruction of complex free energy landscapes by multiple walkers metadynamics. J. Phys. Chem. B 2006, 110, 3533-3539. 
Table 1: Simulation setups.

\begin{tabular}{|c|c|c|}
\hline & $\mathrm{DFT}$ & DFTB \\
\hline Method & PBE-D2 & DFTB3/3ob \\
\hline System & $(\mathrm{PTO})\left(\mathrm{H}_{2} \mathrm{O}\right)_{30}\left(\mathrm{H}^{+}\right)$ & $(\mathrm{PTO})\left(\mathrm{H}_{2} \mathrm{O}\right)_{70}(\mathrm{HCl})$ \\
\hline Box Size & $11.52 \AA$ & $14.93 \AA$ \\
\hline Software & VASP & $\mathrm{DFTB}^{+}$ \\
\hline Metadynamics & \multicolumn{2}{|c|}{$\begin{array}{l}3 \text { CVs: } s_{1}=d, s_{2}=n, s_{3}=\phi \\
\text { V range: }-0.27 \AA<d<2.38 \AA \\
\text { nal hill height: } h=0.2 \mathrm{kcal} / \mathrm{mol} \\
\text { dth: } \sigma_{1}=0.08 \AA, \sigma_{2}=0.033, \sigma_{3}=9^{\circ} \\
\text { deposition: every } 10.6 \text { fs on average } \\
\quad 12 \text { walkers } \\
\text { simulation length: } 250 \mathrm{ps} \times 12 \\
\text { erature: } 573 \mathrm{~K}, \mathrm{MNHC} \text { thermostats } \\
\text { step size: } 0.25 \mathrm{fs} \\
\text { software: } \mathrm{PIMD}^{52}\end{array}$} \\
\hline Blue moon ensemble & \multicolumn{2}{|c|}{$\begin{array}{c}1 \mathrm{CV}: s=d_{\alpha} \\
\text { CV range: } 1.59 \AA \leq d_{\alpha} \leq 5.08 \AA \\
12 \text { constraint points } \\
\text { simulation length: } 133 \mathrm{ps} \times 12 \\
\text { temperature: } 573 \mathrm{~K}, \mathrm{MNHC} \text { thermostats } \\
\text { step size: } 0.25 \mathrm{fs} \\
\text { software: } \mathrm{PIMD}^{52}\end{array}$} \\
\hline
\end{tabular}


Table 2: Atoms relevant to the collective variables.

\begin{tabular}{ccccc}
\hline \hline Pathways & 6 PTO1 & 6 PTO5 & 5PTO2 & 5PTO5 \\
\hline $\begin{array}{c}i \text { atom for } n_{i} \\
j, k \text { atoms for } d=r_{j k}-r_{j i}\end{array}$ & $\mathrm{O}_{6}, \mathrm{O}_{8}$ & $\mathrm{O}_{5}, \mathrm{O}_{6}$ & $\mathrm{C}_{2}, \mathrm{O}_{8}$ & $\mathrm{C}_{5}, \mathrm{O}_{7}$ \\
$l, m$ atoms for $\phi=i-j-l-m$ dihedral & $\mathrm{C}_{2}, \mathrm{C}_{3}$ & $\mathrm{C}_{4}, \mathrm{C}_{3}$ & $\mathrm{C}_{3}, \mathrm{C}_{4}$ & $\mathrm{C}_{4}, \mathrm{C}_{3}$ \\
\hline
\end{tabular}


Table 3: The free energy barrier of PTO in $\mathrm{kcal} / \mathrm{mol}$.

\begin{tabular}{cc|ccc|ccc|c}
\hline \hline \multirow{2}{*}{ Product } & & \multicolumn{3}{|c|}{ PIMD/DFT } & \multicolumn{3}{c|}{ PIMD/DFTB } & exptl. \\
& Path & $\Delta A_{\text {MTD }}$ & $\Delta A_{\text {BME }}$ & $\Delta A_{\text {DFT }}$ & $\Delta A_{\text {MTD }}$ & $\Delta A_{\text {BME }}$ & $\Delta A_{\text {DFTB }}$ & $\Delta A_{\text {exptl. }}$ \\
\hline \multirow{2}{*}{ THFA } & 5PTO2 & $32 \pm 1.0$ & $1.5 \pm 0.5$ & $33 \pm 1.1$ & $31 \pm 1.3$ & $1.7 \pm 0.3$ & $33 \pm 1.3$ & \multirow{2}{*}{41.7} \\
& 5PTO5 & $32 \pm 2.0$ & $1.1 \pm 1.0$ & $33 \pm 2.3$ & $34 \pm 0.8$ & $0.9 \pm 0.3$ & $35 \pm 0.9$ & \\
\multirow{2}{*}{ 3-HTHP } & 6PTO1 & $35 \pm 1.0$ & $4.0 \pm 0.5$ & $39 \pm 1.1$ & $41 \pm 1.8$ & $2.0 \pm 0.4$ & $43 \pm 1.8$ & \multirow{2}{*}{43.9} \\
& 6PTO5 & $36 \pm 1.5$ & $2.8 \pm 0.5$ & $39 \pm 1.6$ & $33 \pm 1.6$ & $3.7 \pm 0.3$ & $37 \pm 1.6$ & \\
\hline
\end{tabular}


<smiles>O[C@H]1CCCOC1</smiles>

\section{R-3HTHP}<smiles>O[C@H]1CCCOC1</smiles>

R-3HTHP
(3)
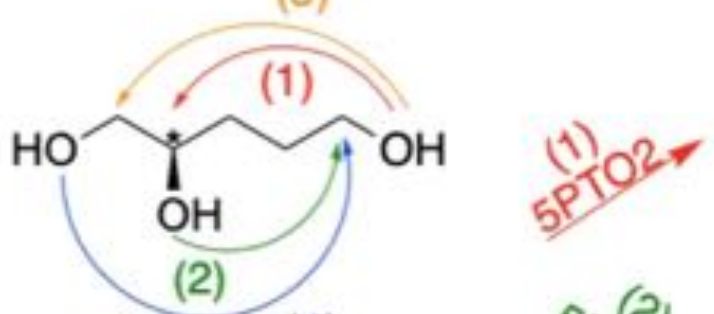<smiles>OC[C@H]1CCCO1</smiles>

S-THFA

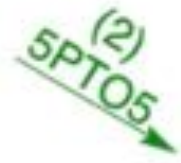

R-THFA

Figure 1: Possible reaction paths from PTO to THFA or 3-HTHP. 

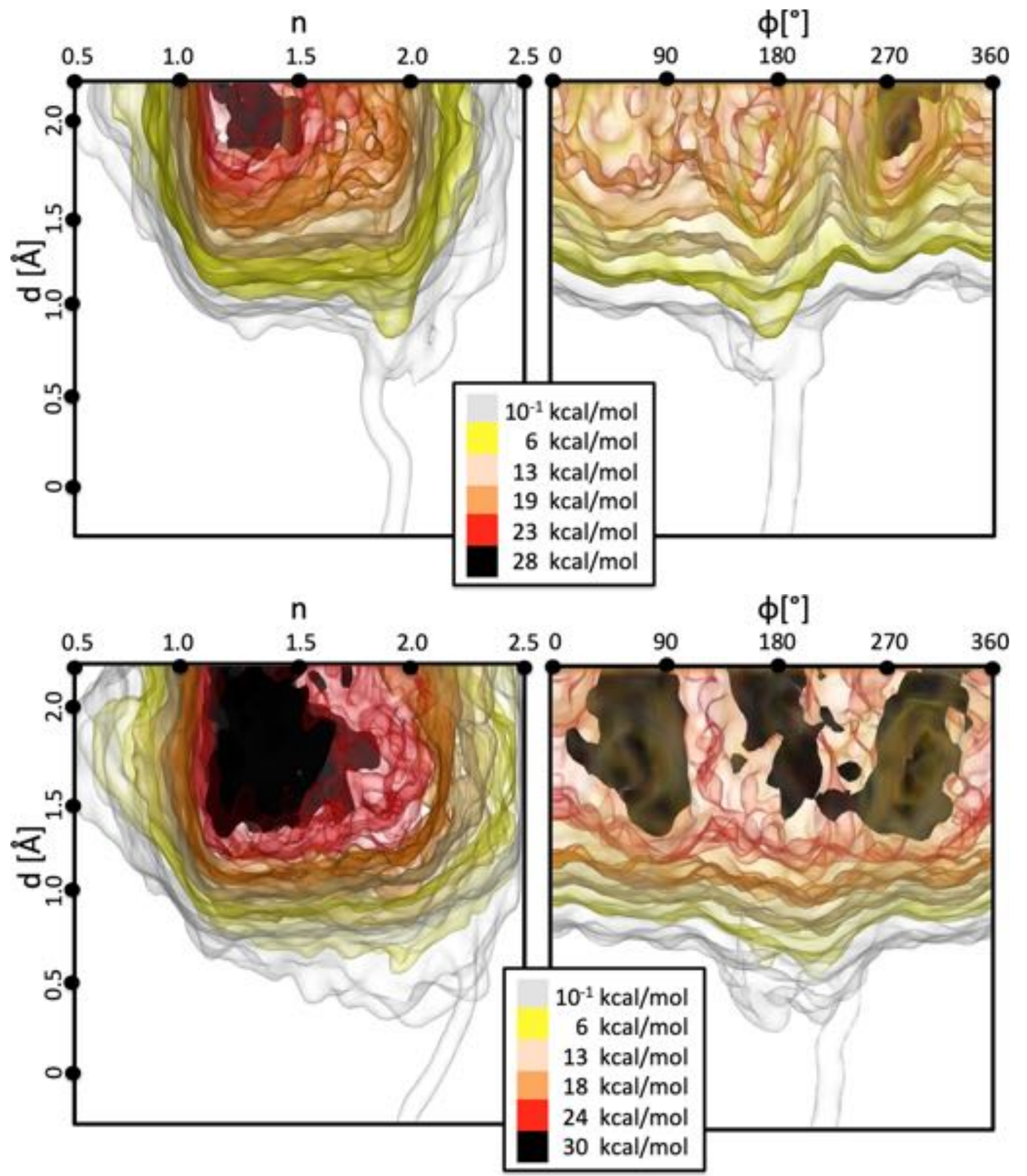

Figure 2: Three-dimensional free energy surface of 5PTO2 (top panels) and 5PTO5 (bottom panels) with respect to $d, \phi$ and $n$, obtained from DFT metadynamics simulations. The side views of free energy surfaces are displayed in a direction perpendicular to $d, n$ (left panels) and parallel to $d, \phi$ (right panels). They are shown by different transparent colors with increasingly leveling contours, from black (confined conformation) to white (TS). 

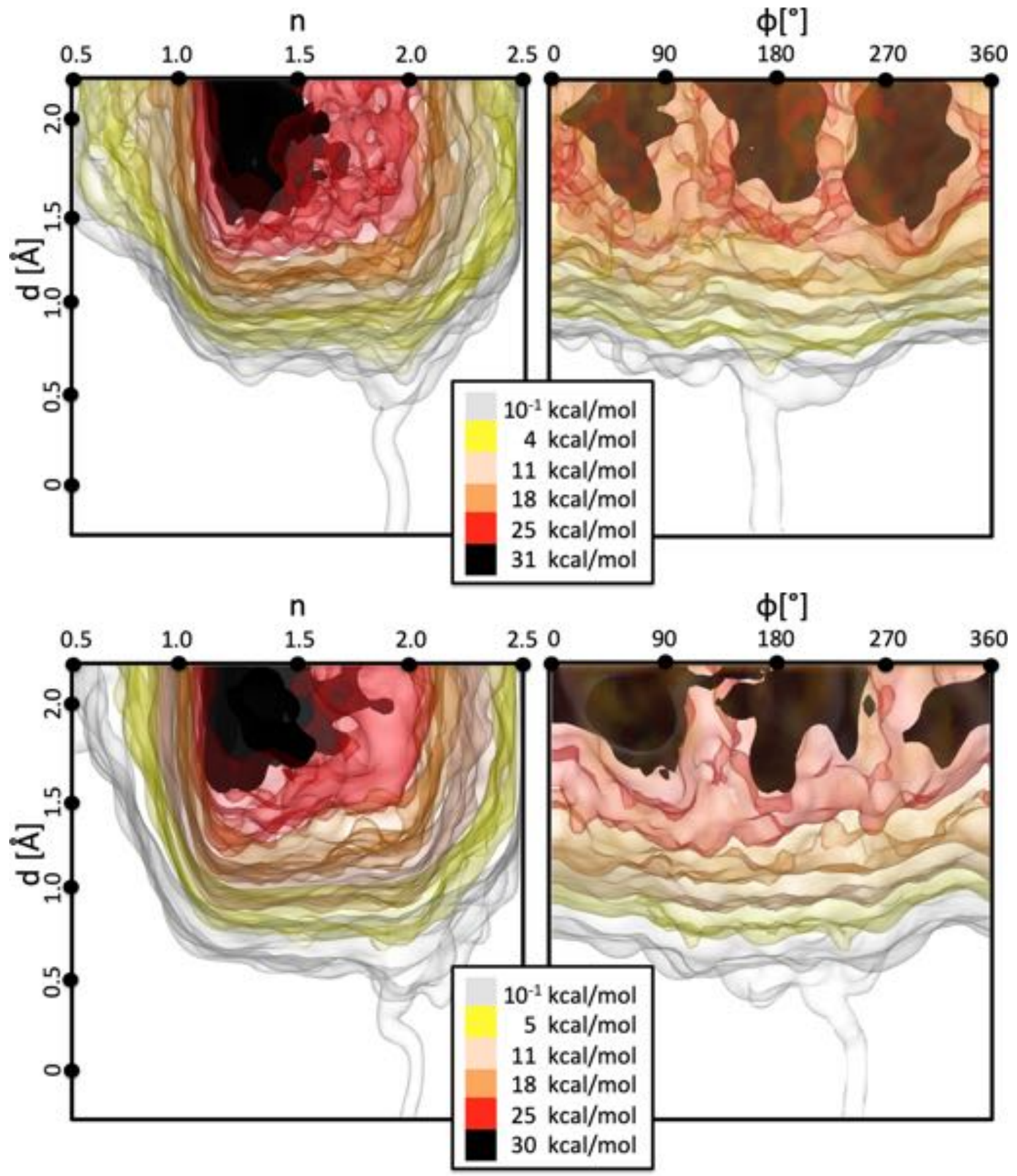

Figure 3: Three-dimensional free energy surface of 6PTO1 (top panels) and 6PTO5 (bottom panels) with respect to $d, \phi$ and $n$, obtained from DFT metadynamics simulations. The side views of free energy surfaces are displayed in a direction perpendicular to $d, n$ (left panels) and parallel to $d, \phi$ (right panels). They are shown by different transparent colors with increasingly leveling contours, from black (confined conformation) to white (TS). 


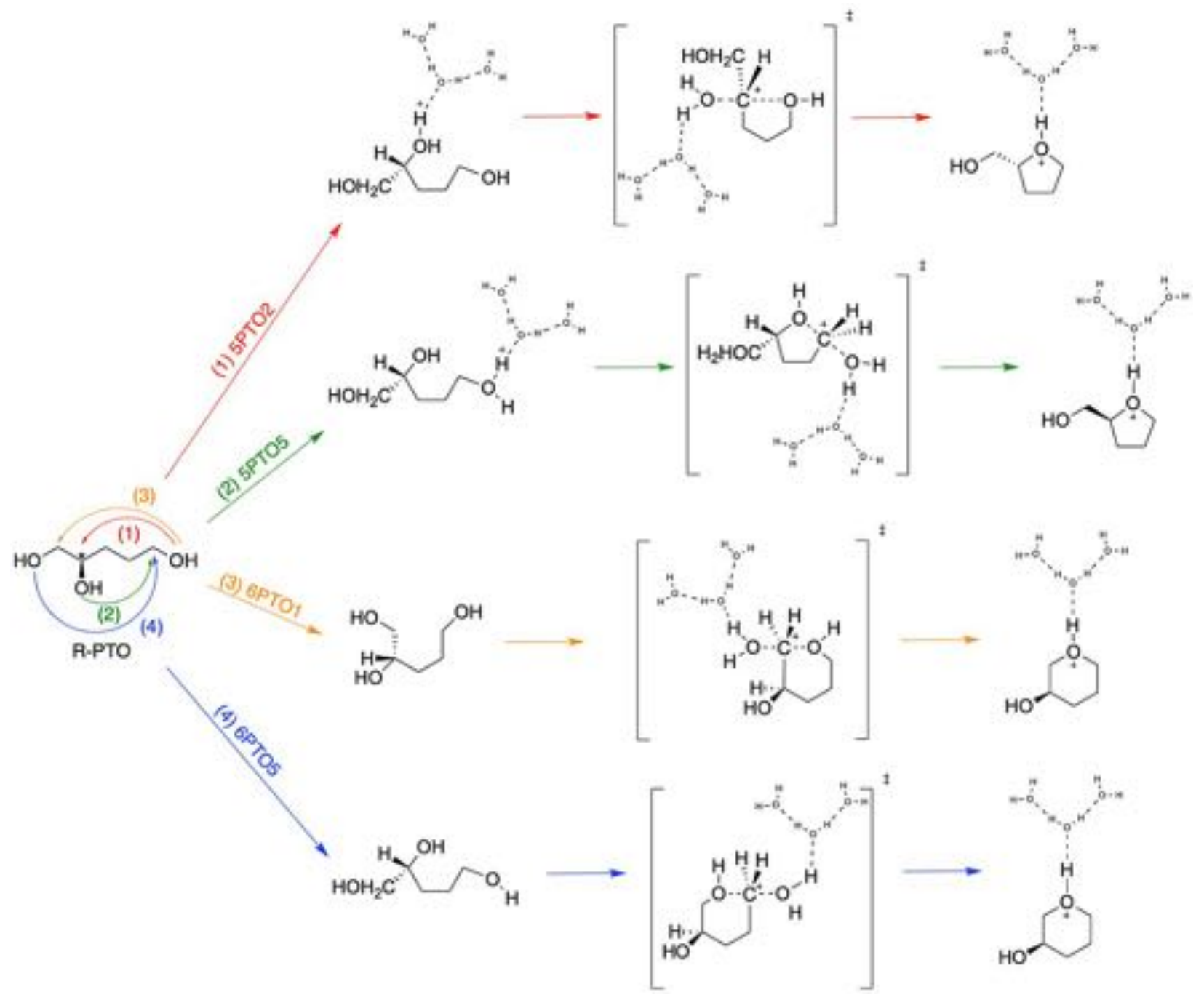

Figure 4: Dehydration pathways of PTO in hot water obtained from metadynamics simulations. 


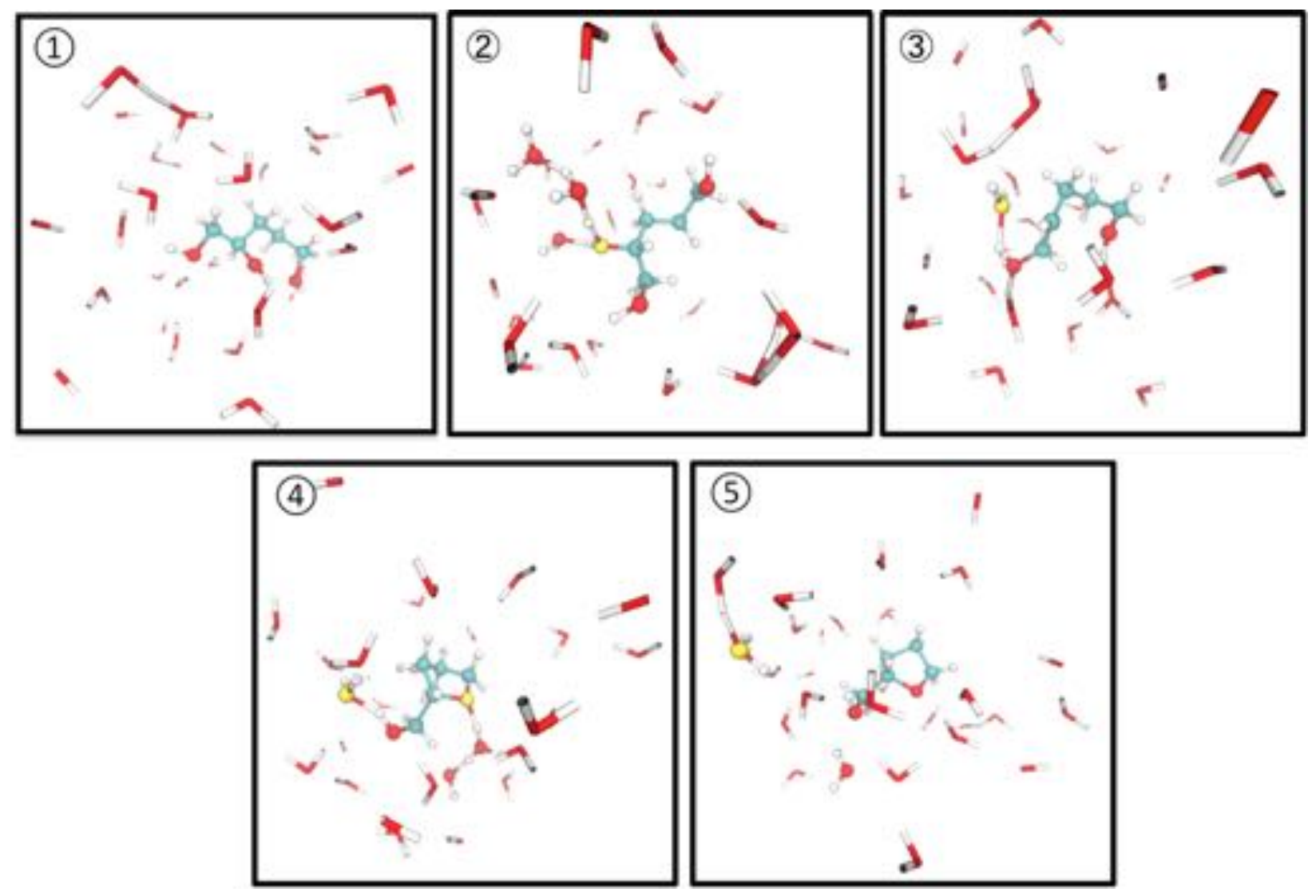

Figure 5: Typical molecular configurations along the 5PTO2 pathway from DFT metadynamics. (1) PTO is in its confined configuration. (2) Protonation of the hydroxyl group occurs. (3) Transition state of PTO in 5PTO2 pathway. (4) The proton of protonated cyclic ether is released into surrounding water molecules. (5) 3-HTHP product is formed. The oxygen of protonated hydroxyl group is colored in yellow. 

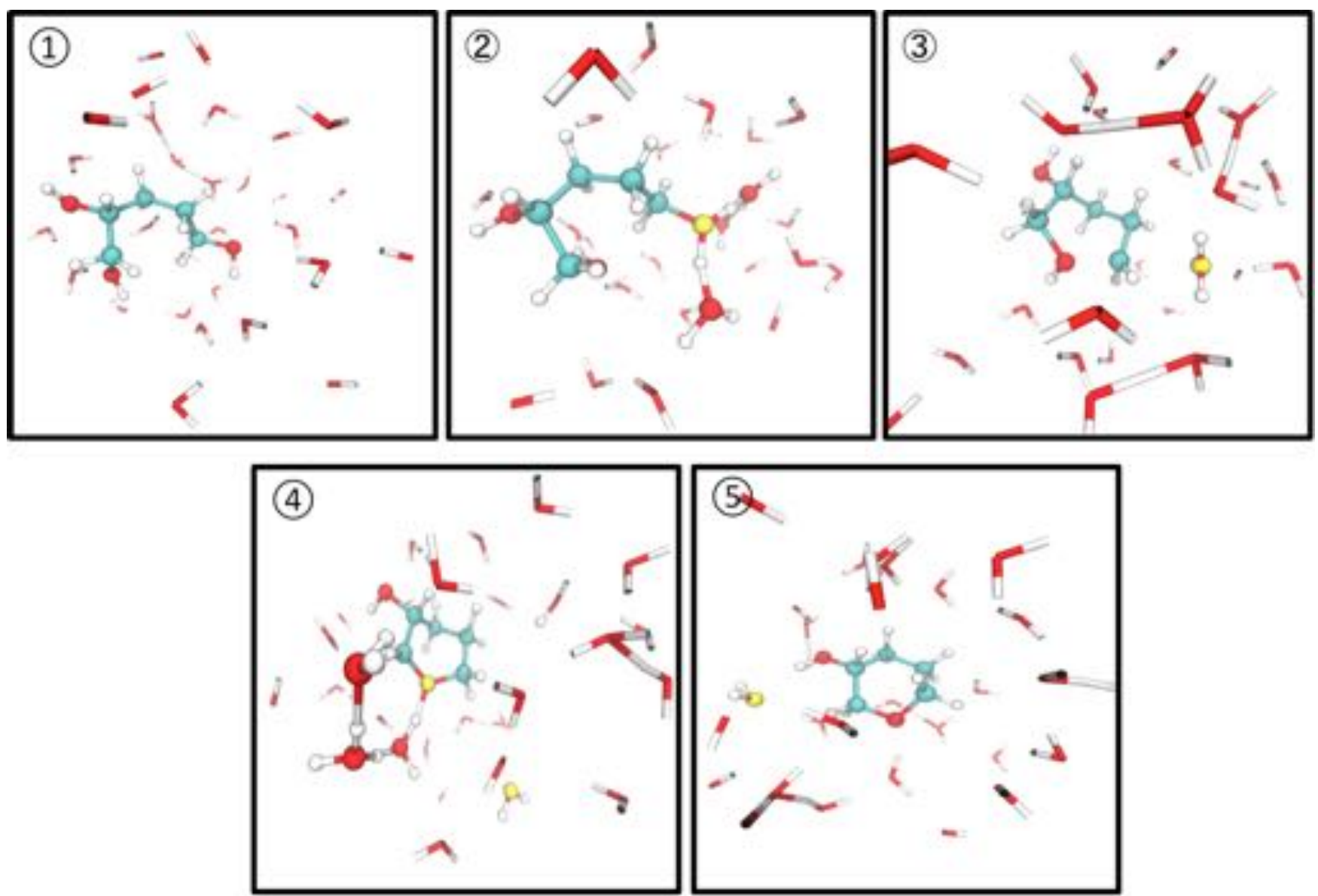

Figure 6: Typical molecular configurations along the 6PTO5 pathway from DFT metadynamics. (1) PTO is in its confined configuration. (2) Protonation of the hydroxyl group occurs. (3) Transition state of PTO in 6PTO5 pathway. (4) The proton of protonated cyclic ether is released into surrounding water molecules. (5) THFA Product is formed. The oxygen of protonated hydroxyl group is colored in yellow. 


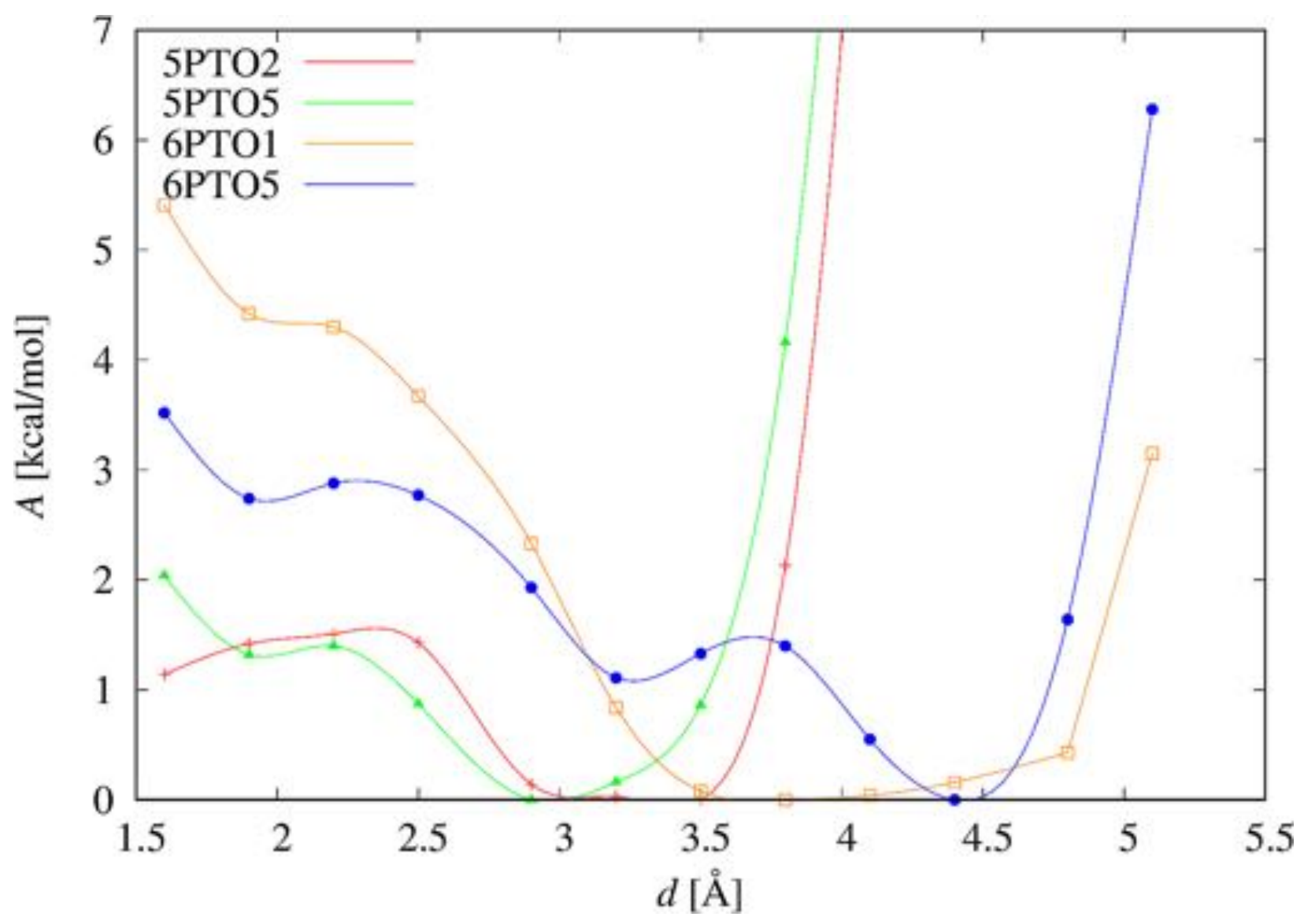

Figure 7: The free energy curves of different paths obtained from DFT blue moon ensemble simulations. 


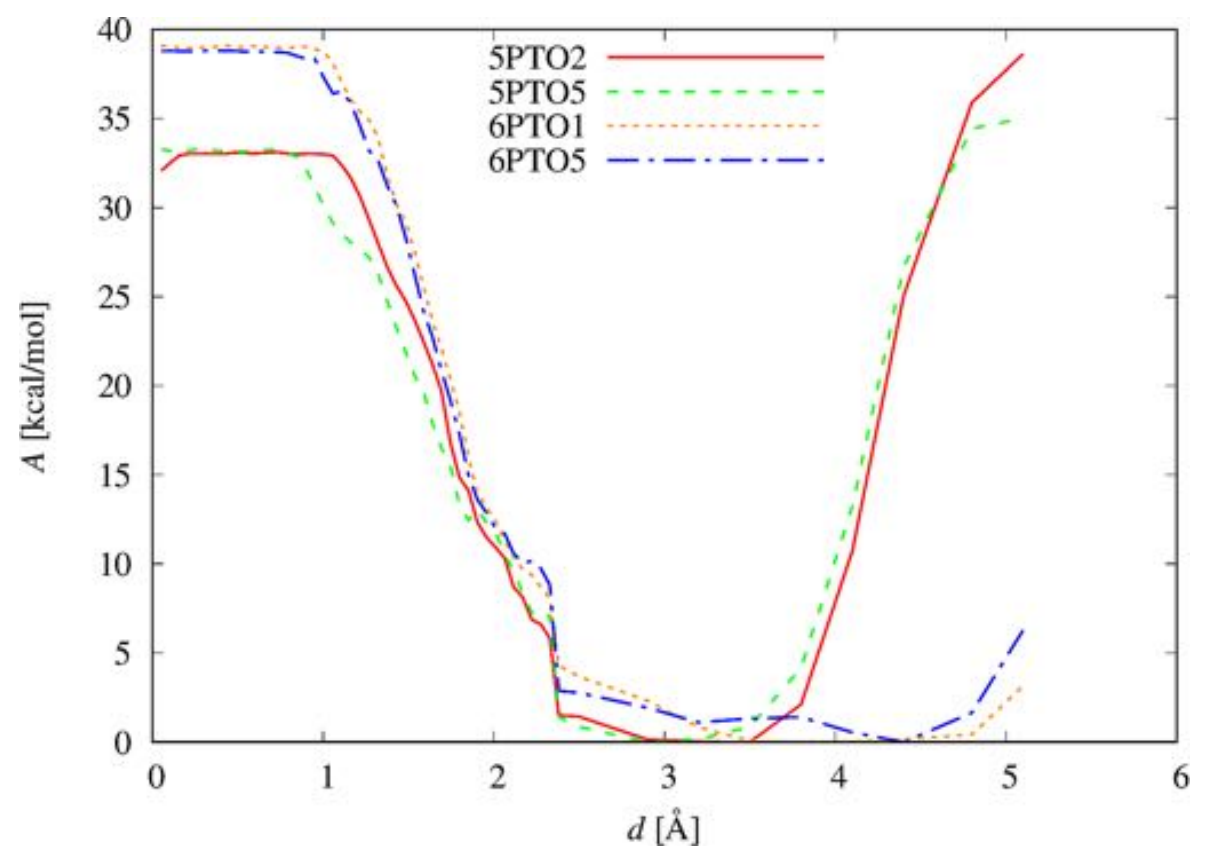

(a)

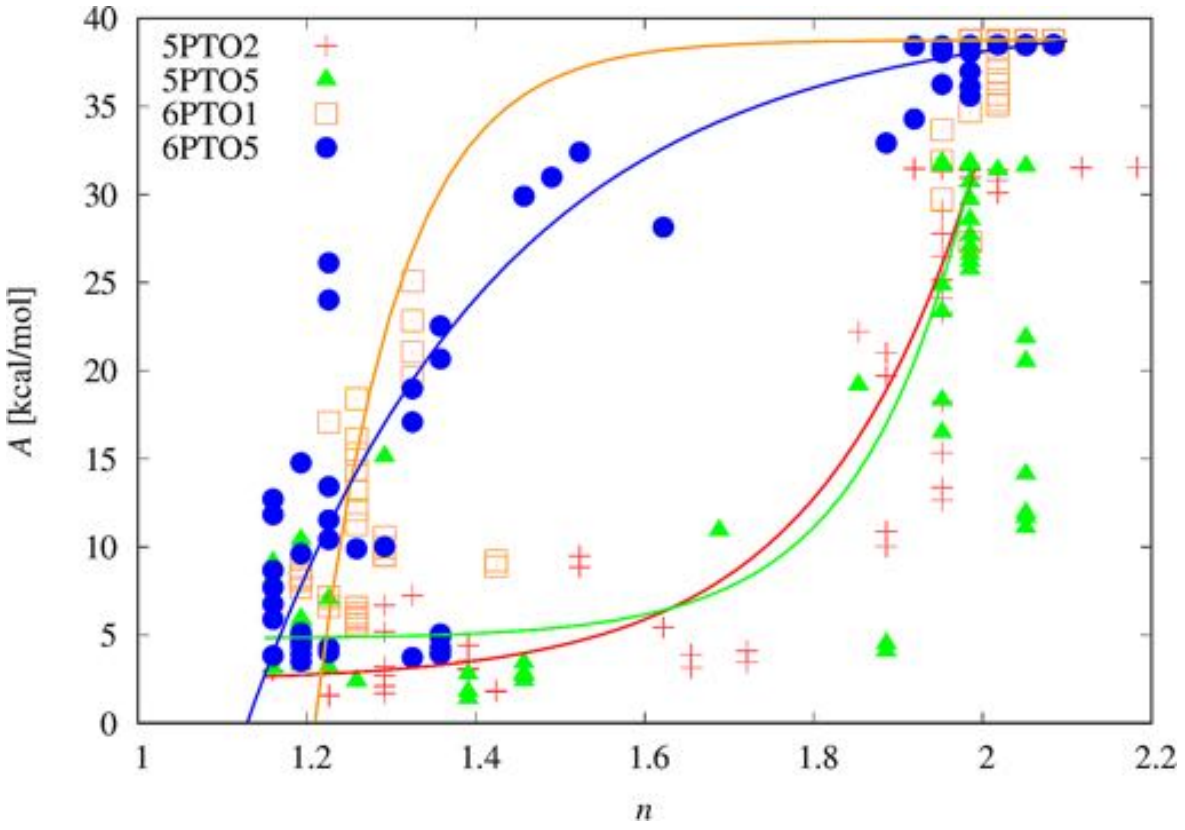

(b)

Figure 8: Free energy curves along the minimum free energy path depicted as functions of $d$ (top panel) and $n(d)$ (bottom panel) obtained from DFT MTD simulations. The curves in (a) are connected to the free energy curves obtained from DFT blue moon ensemble simulations at $d=2.38 \AA$. The lines shown as a guide to the eye are least-square fit to function $a \exp (b x)+c$. 


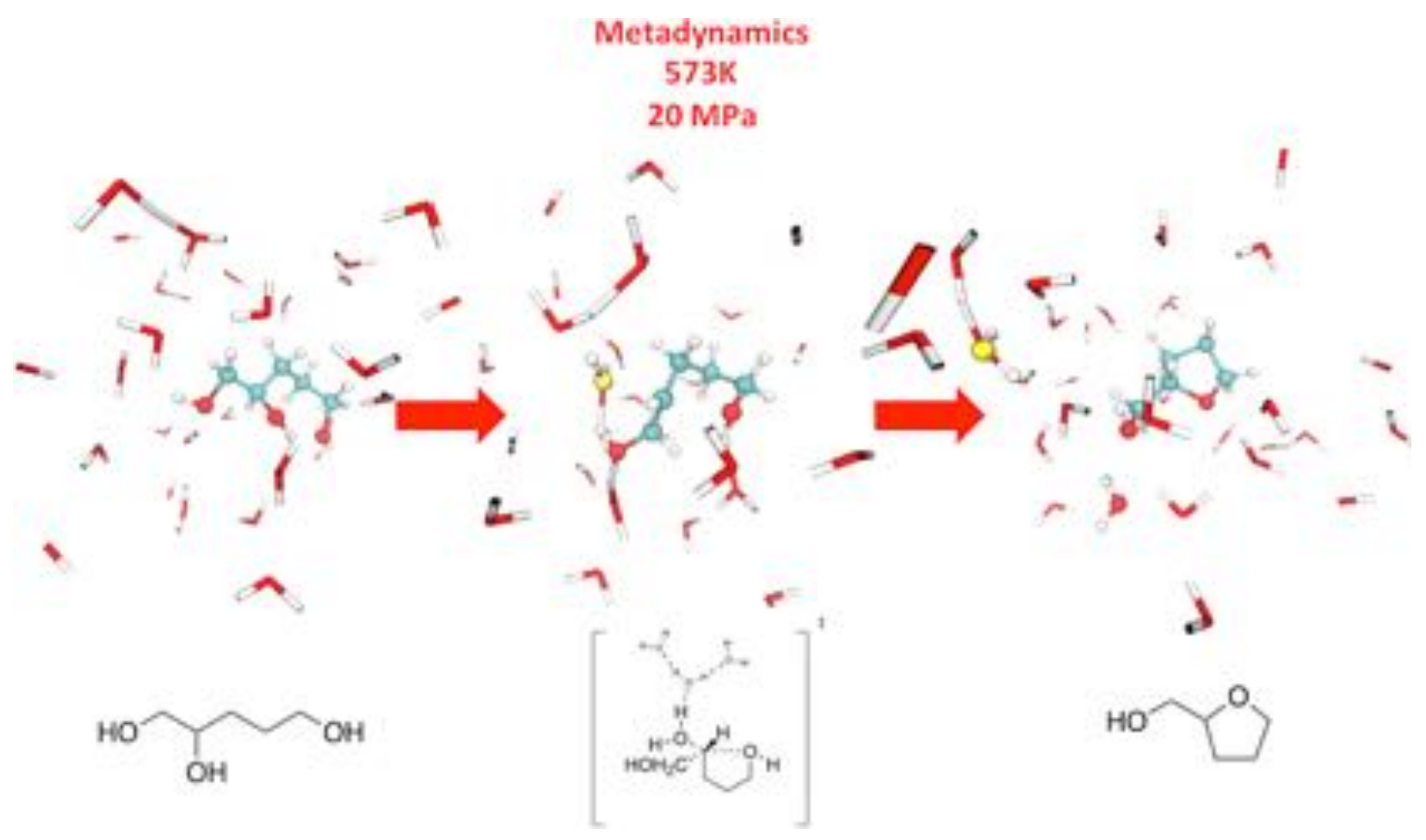

